Ann. Biol. anim. Bioch. Biophys., 1978, 18 (6), 1333-1342.

\title{
Analyse biométrique de l'index de marquage des cellules folliculeuses et de la taille des ovocytes des follicules primordiaux d'ovaire de ratte adulte cyclique
}

\author{
par J. C. MARIANA
}

avec la collaboration technique de Marie-José MANICOM

Station de Physiologie de la Reproduction, I.N.R.A., Nouzilly, 37380, Monnaie.

Summary. Follicle cell labelling index as related to primordial follicle oocyte size. Biometric analysis in cycling adult female rat.

The incorporation of tritiated thymidine into the nuclei of granulosa cells was studied in ovarian follicles of cycling adult rats. It was found that : in follicles having less than 35 granulosa cells, the proportion of follicles labelled increased as the number of cells per follicle augmented; in follicles having more than 35 granulosa cells, the labelling index did not vary throughout the cycle ; the theoretical proportion of follicles having at least one labelled granulosa cell, and calculated from the labelling index, was higher than that actually observed. When the analysis was considered in relation to the size of the oocyte, it was suggested that an oocyte factor was probably responsible for the recommencement of follicular growth ; thus, follicles with an oocyte larger than $512 \mu^{2}$, and having more than 15 cells, were all labelled. This report proposes that the primordial follicle population is heterogeneous and that the pool of primordial follicles cannot be reduced to those which have only 4 or 5 follicle cells; an upper limit of this number in the rat may be 35 cells for primordial follicles.

\section{Introduction.}

La croissance des follicules chez la ratte immature et la ratte cyclique a été particulièrement étudiée par Pedersen (1970) au moyen de la thymidine tritiée ; cette étude porte essentiellement sur les follicules ayant plus de 40 cellules folliculeuses, or it est indispensab e, pour analyser le renouvellement de la population des follicules en croissance, de connaître la probabilité de démarrage de la croissance d'un follicule primordial (Mariana ef Millier, 1977) ; nous avons examiné l'index de marquage des cellules de follicules ayant moins de 40 cellules folliculeuses chez des rattes adultes cycliques à différents moments du cycle ; sur la base de cet index de marquage, nous avons tenté de préciser si les follicules primordiaux constifuent une réserve homogène à partir de laquelle certains d'entre eux commencent leur croissance. 


\section{Matériel et méthodes.}

Protocole expérimental.

L'étude a porté sur 16 rattes Wistar adultes et cycliques de 3 mois ayant des cycles normaux de 4 jours contrôlés par frottis vaginaux, et soumises à un régime journalier de $14 \mathrm{~h}$ de lumière et $10 \mathrm{~h}$ d'obscurité. L'alimentation a été maintenue constante.

On a injecté aux animaux de la thymidine tritiée, à raison de $2 \mu \mathrm{Ci} / \mathrm{g}$ de poids vif ; les animaux étaient répartis en 4 lots, le premier lot traité le matin de l'œstrus à $9 \mathrm{~h}$, le second $6 \mathrm{~h}$ après, le troisième $12 \mathrm{~h}$ après, etc... L'intervalle entre chaque traitement étant ainsi de $6 \mathrm{~h}$ sur l'ensemble d'un cycle. Les ovaires ont été prélevés $1 \mathrm{~h}$ après l'injection et fixés; un ovaire sur deux, pris au hasard, est analysé.

Les coupes de $5 \mu$ d'épaisseur montées en série sont colorées au Feulgen recouvertes d'émulsion Illford $K_{2}$ et révélées après une exposition d'une durée de 3 semaines.

Pour chaque ovaire analysé, l'ensemble des follicules ayant moins de 40 cellules de granulosa a été dénombré et pour chaque follicule, les cellules marquées ef non marquées ont été comptées dans la coupe où la nucléole de l'ovocyte est présent.

On appelle follicule marqué un follicule ayant au moins une cellule de granulosa marquée.

Sur un échantillon de 260 follicules, nous avons mesuré la taille de l'ovocyte sur la coupe où le nucléole est visible et compté sur cette même coupe le nombre de cellules folliculeuses.

Nous avons calculé la taille en $\mu^{2}$ de l'ovocyte à partir de la mesure de deux diamètres rectangulaires estimée au moyen d'un réticule oculaire.

Méthode d'analyse du pourcentage des cellules folliculeuses qui ont démarré un cycle cellulaire.

Définition. - La croissance du follicule commence quand tout ou partie des cellules folliculeuses ont entamé un cycle cellulaire. Cette définition est consistante avec celle de la croissance du follicule par multiplication des cellules folliculeuses.

Dans une population de cellules en multiplication non synchronisée, la probabilité qu'une cellule soit marquée en phase $S$ est :

$$
P=e^{\frac{t_{2}}{T} \cdot \ln 2}\left[e^{\frac{t_{s}}{T} \cdot \ln 2}-1\right]
$$

où $t_{S}, t_{2}$ sont respectivement la durée des phases $S$ et $G_{2}$ et $T$ la longueur du cycle cellulaire (Cleaver, 1965). $\mathrm{p}$ est également appelé index de marquage.

En supposant que les $n$ cellules d'un follicule dont la croissance a débuté, reprennent ensemble leur cycle cellulaire, mais de façon indépendante, on peut estimer la probabilité $P_{(i)}$ qu'une cellule d'un follicule à i cellules soit en phase $S$, (index de marquage) par :

$$
P_{(i)}=\frac{\sum_{J=2}^{i} J \cdot N_{J}^{(i)}}{i \cdot \sum N_{J-1}^{(i)}-N_{1}^{(i)}}
$$

où $\mathrm{N}_{J}^{(i)}$ est le nombre de follicules ayant i cellules dont $\mathrm{j}$ marquées. 
Les follicules sont classés en huit catégories selon le nombre de cellules entourant l'ovocyte ( 1 à 5,5 à 10,10 à 15,15 à 20,20 à 25,25 à 30,30 à 35,35 à 40 ) ; ce découpage est arbitraire mais il permet d'avoir suffisamment de follicules par classe, et il est assez fin pour que les variations de $P_{(i)}$ à l'intérieur d'une classe soient inférieures à celles qui peuvent exister entre les classes.

Nous avons également fait l'hypothèse que $P_{(i)}$ était identique pour tous les follicules d'une catégorie.

Pour l'étude de marquage en fonction du cycle, celui-ci a été divisé en 4 périodes de $24 \mathrm{~h}$ et les animaux regroupés dans ces 4 périodes selon l'heure et le moment d'injection.

\section{Résultats.}

La figure 1 représente l'histogramme du nombre moyen des follicules marqués et non marqués selon le nombre des cellules qui entourent l'ovocyte.

Dans l'histogramme, les résultats de l'ensemble des 16 rattes étudiées ont été regroupés.

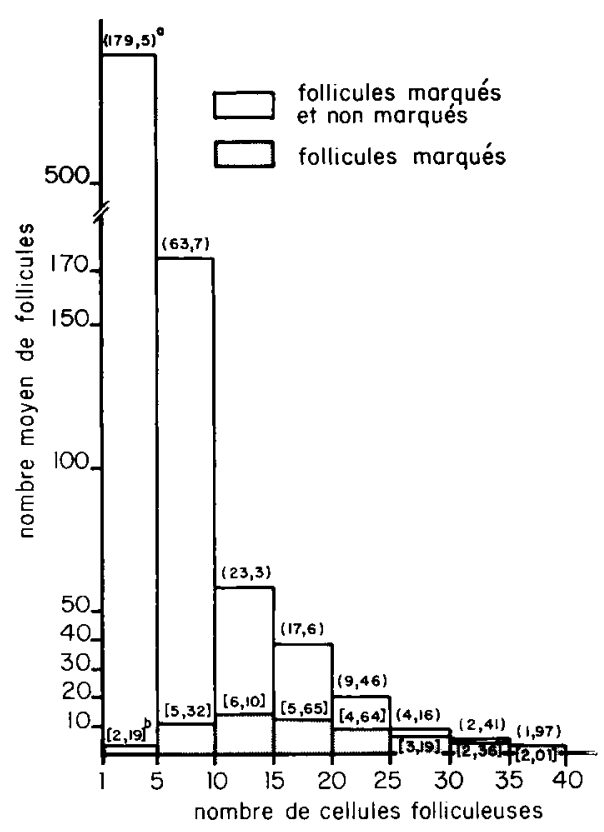

FIG. 1.

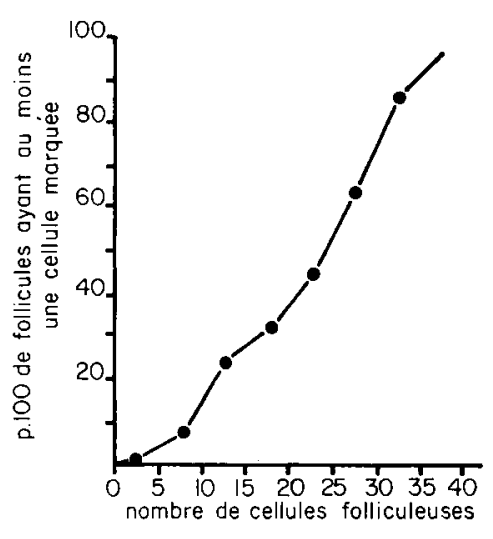

FIG. 2 .

FIG. 1. - Hisiogramme du nombre de follicules en fonction du nombre de cellules folliculeuses.

( ) a écart type du nombre de follicules non marqués de chaque classe,

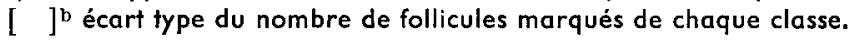

FIG. 2. - Evolution du p. 100 de follicules ayant au moins une cellule marquée avec le nombre de cellules folliculeuses. 
On observe que :

- le nombre total des follicules décroît quand le nombre des cellules folliculeuses croît ;

- quel que soit le nombre des cellules qui entourent l'ovocyte, il existe toujours des follicules qui ont au moins une cellule marquée;

- le nombre des follicules avec au moins une cellule marquée croît, passe par un maximum pour les follicules ayant de 11 à 15 cellules folliculeuses, et décroît ensuite; - le pourcentage de follicules marqués croît progressivement en fonction du nombre des cellules folliculeuses (fig. 2, tabl. 2) ;

- il n'existe pas de follicules ayant plus de 35 cellules dont au moins une cellule folliculeuse ne soit marquée.

Nous avons ensuite analysé l'influence du moment du cycle ef du nombre de cellules folliculeuses sur l'index de marquage.

\section{TABLEAU 1}

Index de marquage $(\times 100)$ en fonction du jour du cycle et de la classe des follicules

\begin{tabular}{|c|c|c|c|c|}
\hline \multirow{2}{*}{$\begin{array}{l}\text { Classe des follicules } \\
\text { (nombre de cellules folliculeuses) }\end{array}$} & \multicolumn{4}{|c|}{ Jour du cycle } \\
\hline & 1 & II & III & IV \\
\hline $\begin{array}{c}1-5 \\
6-10 \\
11-15 \\
16-20 \\
21-25 \\
26-30 \\
31-35 \\
36-40\end{array}$ & $\begin{array}{l}0 \\
4,8 \\
5,6 \\
7,8 \\
8,5 \\
8,98 \\
10,3 \\
12,7\end{array}$ & $\begin{array}{r}0 \\
2,0 \\
7,2 \\
3,2 \\
6,8 \\
6,0 \\
11,4 \\
10,4\end{array}$ & $\begin{array}{c}0 \\
7,0 \\
7,1 \\
11,4 \\
9,21 \\
12,9 \\
11,3 \\
13\end{array}$ & $\begin{array}{r}0 \\
5,5 \\
7,4 \\
7,3 \\
8,5 \\
11,1 \\
11,5 \\
9,4\end{array}$ \\
\hline
\end{tabular}

TABLEAU 2

Nombre de follicules d'une classe (i) et ayant (j) cellules marquées

\begin{tabular}{crrrrrrr}
\hline & \multicolumn{7}{c}{ Nombre de cellules marquées (i) } \\
\cline { 2 - 8 } Classe (i) & 0 & 1 & 2 & 3 & 4 & 5 & $>5$ \\
\hline $1-5$ & 8240 & 49 & 2 & & & & \\
\hline $6-10$ & 2617 & 142 & 27 & 5 & & & \\
\hline $11-15$ & 716 & 147 & 42 & 22 & 5 & 1 & 1 \\
\hline $16-20$ & 442 & 101 & 48 & 23 & 10 & 5 & 3 \\
\hline $21-25$ & 181 & 53 & 31 & 27 & 16 & 6 & 1 \\
\hline $26-30$ & 53 & 24 & 21 & 20 & 11 & 3 & 13 \\
\hline $31-35$ & 12 & 12 & 11 & 11 & 10 & 9 & 15 \\
\hline $36-30$ & 2 & 2 & 2 & 11 & 11 & 1 & 10 \\
\hline
\end{tabular}


Le tableau 1 regroupe les valeurs moyennes de $P_{(i)}$ pour les quatre périodes du cycle et les huit catégories de follicules.

L'analyse de variance réalisée sur les données transformées par la transformation Arcsin $\sqrt{P_{(i)}}$ révèle que $P_{(i)}$ est significativement différent selon les classes de follicules considérés, mais ne varie pas significativement au cours du cycle pour chacune des classes de follicules ; l'index de marquage croît proportionnellement au nombre de cellules folliculeuses, et la pente de la droite de régression correspondant aux quatre périodes du cycle n'est pas différente d'une période à l'autre, avec un risque inférieur ou égal à 1 p. 100, la valeur de la pente commune est 0,251 , donc quand on passe d'une classe de follicule à la suivante l'index de marquage augmente de 1,25 p. 100 (fig. 3).
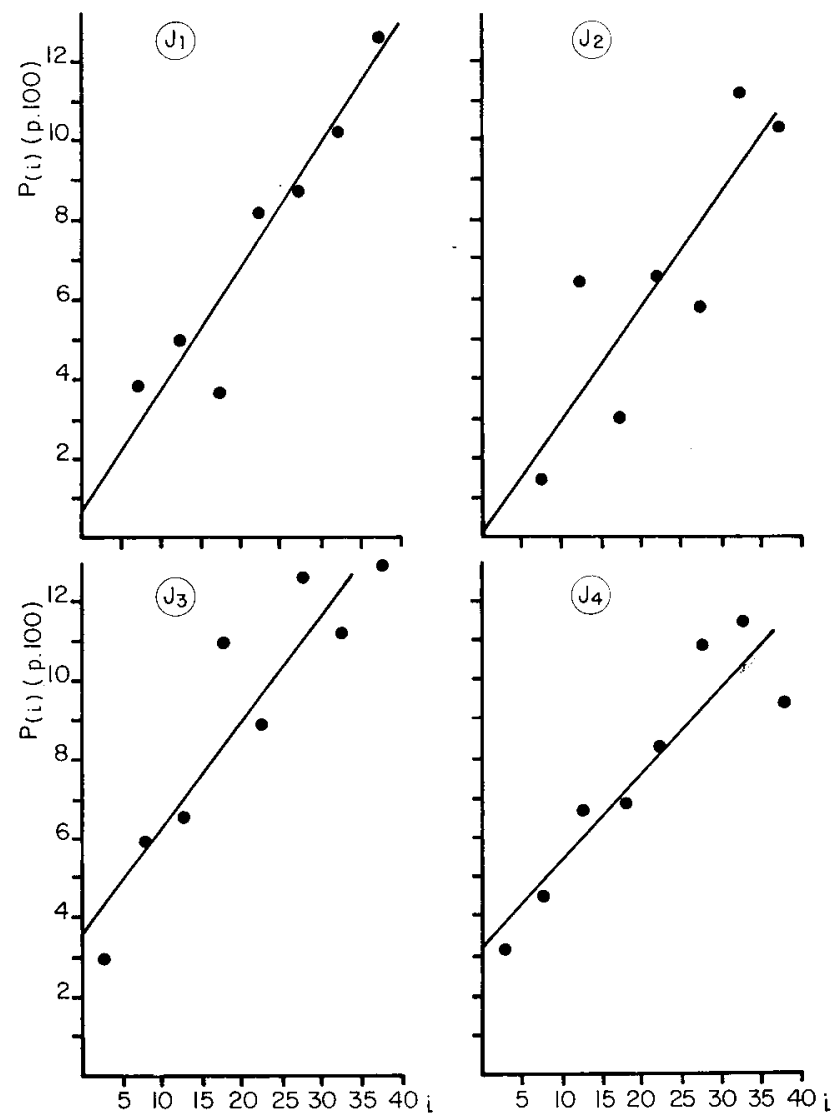

FIG. 3. - Evolution de l'index de marquage $\mathbf{P}_{(\mathrm{i})}$ en fonction du nombre de cellules folliculeuses (i) selon le jour du cycle estrien $\mathrm{J}_{1}, \mathrm{~J}_{2}, \mathrm{~J}_{3}, \mathrm{~J}_{4}$.

Pourcentage de follicules non marqués. - Avec les hypothèses précédentes, la proportion de follicules non marqués en croissance ef qui ont $i$ cellules folliculeuses, est : $\left(1-P_{(i)}\right)^{i}=Q_{(i)}$. On peut donc, pour chaque classe de follicules, calculer un 
pourcentage théorique $Q_{(i)}$ de follicules non marqués qui ont démarré leur croissance. On observe (fig. 4) que $Q_{(i)}$ décroît en fonction de i, mais qu'il reste inférieur au rapport du nombre des follicules non marqués sur le nombre des follicules totaux pour une classe donnée, et quelle que soit la période du cycle considérée.
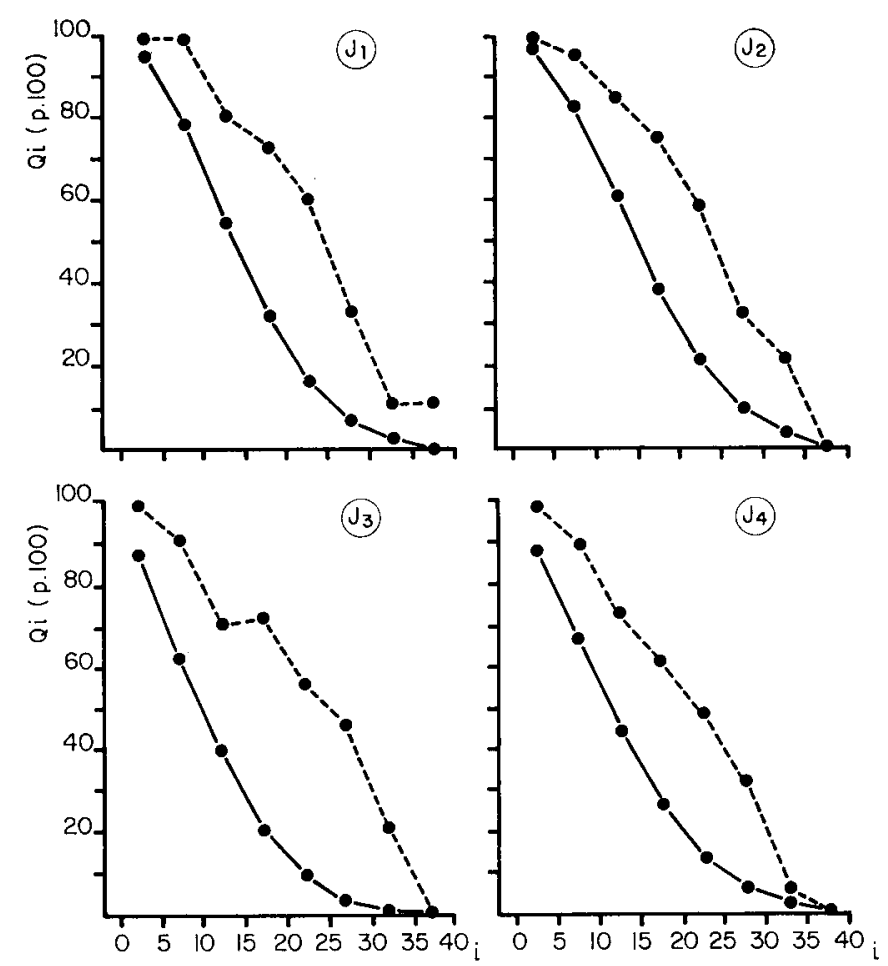

FIG. 4. - Proportion de follicules non marqués $Q_{(i)}$ en fonction du nombre (i) de cellules folliculeuses.

Taille de l'ovocyte et nombre de cellules folliculeuses marquées et non marquées. On a distingué la population des follicules ayant au moins une cellule marquée de celle des follicules n'en ayant pas.

Pour les deux populations, la corrélation entre le nombre de cellules et la taille de l'ovocyte est positive et significative. Les droites de régression du nombre de cellules folliculeuses i sur la taille de l'ovocyte sont respectivement pour les follicules marqués et non marqués : $i=0,00359$ y $+15,09$ et $i=0,0048 y+15,13$.

Les différentes méthodes d'analyse discriminante utilisées ne permettent pas de réaliser un classement a posteriori des follicules avec moins de 40 p. 100 d'erreurs ce qui signifie que, un classement $a$ posteriori des follicules en utilisant une fonction discriminante, est réalisé avec 40 p. 100 d'erreurs. Il semble que le critère taille de l'ovocyłe ait cependant un pouvoir séparateur supérieur à celui du nombre des cellules. On remarque en effet, que la moyenne de la taille des ovocytes de follicules marqués est 
significativement supérieure à celle des ovocytes de foliculles non marqués (fig. 5). La population des points représentant follicules marqués et non marqués forme deux nuages très peu inclinés sur l'axe des failles des ovocytes (fig. 6) ; ces deux nuages ont

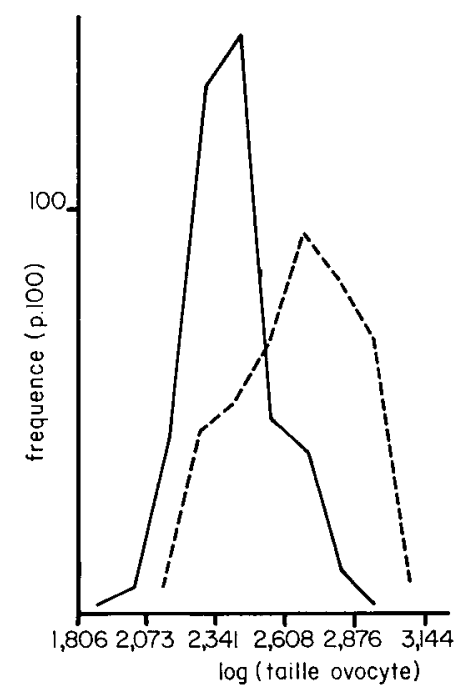

FIG. 5. - Distribution du logarithme de la taille des ovocytes.

- ovocytes de follicules sans cellule folliculeuse marquée.

. . . . . - ovocytes de follicules ayant au moins une cellule folliculeuse marquée.

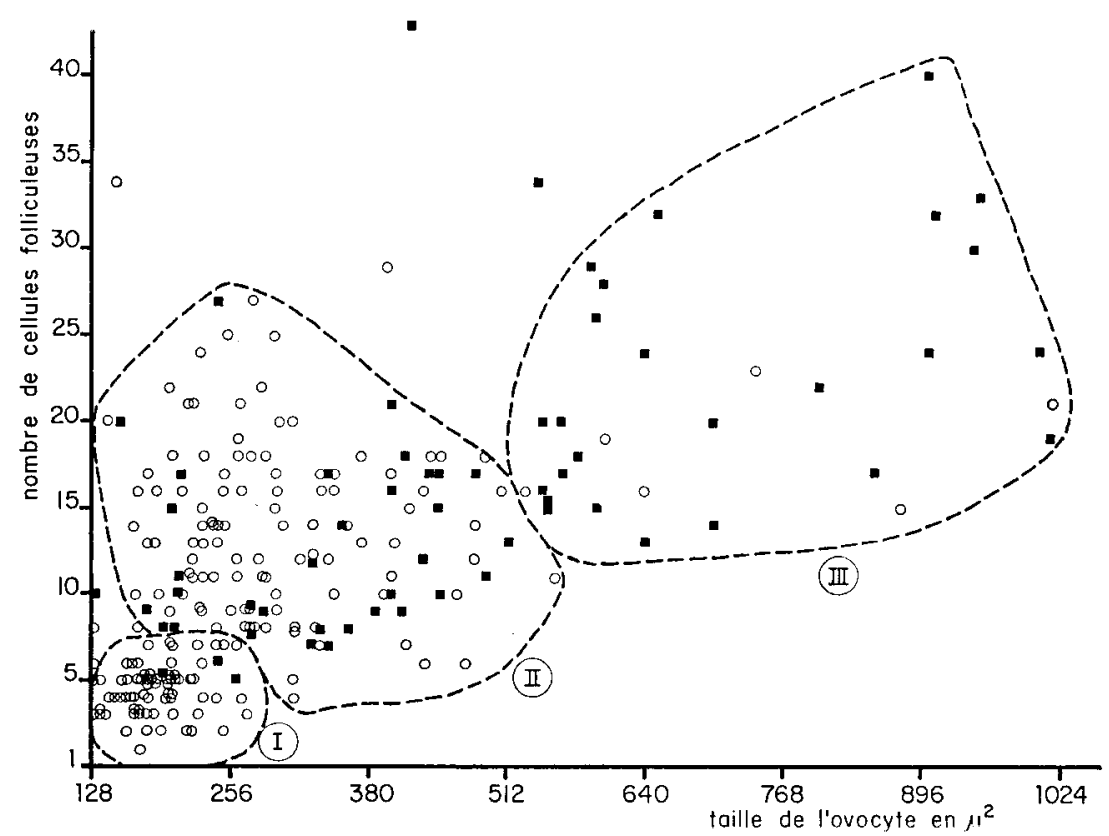

FIG. 6. - Relafion entre la taille de l'ovocyfe et le nombre de cellules folliculeuses des follicules ayant au moins une cellule marquée ( $\mathbf{a})$ et des follicules n'ayant aucune cellule marquée (0). 
une importante partie commune. On peut donc distinguer 3 grands groupes de follicules :

- Groupe 1 : Les follicules dont l'ovocyte a une taille comprise entre 128 et $256 \mu^{2}$ ef de 1 à 6 cellules folliculeuses, très peu sont marqués.

- Groupe 2 : Les follicules dont les ovocytes ont une taille comprise entre 128 et $512 \mu^{2}$ et de 5 à 25 cellules folliculeuses, ce groupe contient des follicules marqués et non marqués.

- Groupe 3 : Les follicules dont l'ovocyte a une faille comprise entre 512 et $1024 \mu^{2}$ et de 12 à 40 cellules folliculeuses, ils sont tous marqués.

Les groupes 1 ef 3 sont clairement séparés par le critère taille de l'ovocyte et moins par celui du nombre des cellules folliculeuses.

Les groupes 1 et 2 sonł par contre séparés par le critère nombre de cellules folliculeuses et peu par la taille de l'ovocyte.

La majorité des follicules que nous avons étudiés ne sont donc pas entrés en croissance et on n'observe pas la relation mise en évidence par Brambell (1928) entre la taille du follicule et la taille de l'ovocyte ; une telle relation peut être établie à partir des follicules du groupe 3.

\section{Discussion.}

Evolution de l'index de marquage en fonction du temps et du nombre des cellules de la granulosa. - Une difficulté essentielle de l'analyse des follicules à moins de 35 cellules folliculeuses, est de nettement séparer la part de la variation potentielle qui existe au niveau des différents paramètres morphologiques, de celle qui est liée à la croissance des follicules.

Nous aboutissons, pour les follicules ayant moins de 40 cellules folliculeuses à des conclusions analogues à celles de Pedersen et Hartman (1971), l'index de marquage croît avec le nombre de cellules folliculeuses. Nous trouvons cependant une valeur légèrement inférieure à celle obtenue par ces auteurs pour les follicules ayant de 20 à 40 cellules, soit 0,10 contre 0,12 . II n'est pas certain que cette faible différence soit significative.

L'index de marquage des follicules à moins de 20 cellules est faible : 0,054, et ceci correspond bien aux observations de Peters ef Levy (1966) sur la faible incorporation de la thymidine tritiée par ces follicules. Quand on passe d'une classe de follicule à la suivante, la variation du pourcentage de follicules marqués et celle du nombre de follicules sont opposées, ceci explique que le nombre de follicules marqués passe par un maximum pour les follicules ayant de 11 à 15 cellules de granulosa.

Proportion de follicules non marqués. - L'écart de signe positif entre les valeurs estimées et les valeurs observées du pourcentage des follicules non marqués est trop élevé pour n'être dô qu'au hasard. Il est nécessaire d'examiner les raisons possibles d'un tel écart.

Les cellules marquées et non marquées d'un follicule sont dénombrées dans la coupe où le nucléole de l'ovocyte est visible ; il est alors possible qu'un follicule soit considéré comme non marqué alors que des cellules marquées existent sur une autre coupe. 
Pour réduire cette erreur d'estimation du pourcentage de follicules non marqués, nous avons utilisé des coupes sériées, et estimé indépendamment les pourcentages pour les 4 rattes d'une même période ; de plus, la probabilité d'une telle erreur diminue avec le nombre des cellules folliculeuses.

Cette source d'erreur n'explique certainement pas à elle seule des écarts pouvant dépasser 20 p. 100 . Bien qu'il soit difficile de suivre un follicule ayant moins de 30 cellules folliculeuses d'une coupe sur l'autre, car la structure du petit follicule est alors peu reconnaissable, nous avons observé que moins de 10 follicules sur 100 étaient marqués sur une section de follicule autre que celle où le nucléole de l'ovocyte était présent.

Nous avons fait l'hypothèse que, dans un follicule démarrant sa croissance, toutes les cellules reprenaient simultanément leur croissance et non obligatoirement dans la même phase du cycle cellulaire.

On peut cependant faire un certain nombre de remarques à ce sujet, permettant d'expliquer les écarts observés.

1) L'estimation du pourcentage des cellules folliculeuses qui entrent en phase de multiplication quand le follicule démarre sa croissance ou qui cessent de se multiplier en cours de croissance du follicule est difficile (Mendelsohn, 1962 ; Hartman et Pedersen, 1970).

2) Les cellules folliculeuses des follicules de moins de 40 cellules auraient un cycle très particulier (Peters et Levy, 1966) encore plus long que celui calculé par Hartman et Pedersen pour les follicules ayant entre 20 et 60 cellules (Epifanova et Terskikh, 1969).

Il est également difficile d'exclure dans ces conditions la possibilité d'un synchronisme partiel des multiplications des cellules folliculeuses.

Enfin, le rôle de l'atrésie est certainement prépondérant. Ingram (1962) avait déjà signalé qu'il était extrêmement difficile de définir des critères précis et irréfutables de l'atrésie des petits follicules. Il est donc possible qu'une proportion importante des follicules qui n'incorporent pas la thymidine tritiée soient en début d'atrésie.

Une autre source d'hypothèse possible concerne la croissance de l'ovocyte en relation avec le nombre de cellules folliculeuses.

Le fait que les follicules des groupes 1 et 3 sont clairement séparés par la faille de l'ovocyte et moins par le nombre des cellules folliculeuses, semble confirmer :

1) que le démarrage de la croissance des follicules est associé à un accroissement de la taille de l'ovocyie ;

2) que les follicules marqués ont plutôt un gros ovocyte, et qu'il existe un facteur d'initiation de la croissance dans les follicules à gros ovocyte.

Le pool des follicules primordiaux mobilisables serait constitué par des follicules ayant entre 5 et 25 cellules folliculeuses et un ovocyte dont la taille est comprise entre 128 ef $512 \mu^{2}$ (groupe 3).

Les très petits follicules (groupe 1) constituent une population particulière très peu mobilisable : leur origine morphogénétique serait différente de celle des follicules des classes plus élevées )Jeppesen, 1978) ; les ovocytes de ces petits follicules auraient été formés plus tardivement pendant la période fœtale (Mauléon, 1975 ; Kennely et Foote, 1966). 


\section{Conclusion.}

La croissance des follicules à partir du pool est un phénomène continu depuis la naissance de la femelle; la probabilité qu'un follicule reprenne sa croissance croît avec le nombre de cellules qui entourent l'ovocyte et, pour un nombre de cellules données, avec la taille de l'ovocyte. Les follicules ayant plus de 15 cellules et un ovocyte de plus de $512 \mu^{2}$ sont tous marqués, donc tous en croissance.

Reçu en mai 1978.

Accepté en juin 1978.

\section{Références}

BRAMBELL F.W. R., 1928. The development and morphology of the gonads of the mouse. 3 . The growth of the follicles. Proc. roy. Soc. B, 103, 258-272.

CLEAVER J. S., 1965. The relationship between the duration of the $S$ phase and the fraction of cells which incorporate ${ }^{3} \mathrm{H}$-thymidine during exponential growth. Exp. Cell Res., 39, 697-700.

EPIFANOVA O. I., TERSKIKH V. V., 1969. On the resting period in the cell life cycle. Cell. Tiss. Kinet., 2, 75-93.

HARTMAN N. R., PEDERSEN T., 1970. Analysis of the kinetics of granulosa cell populations in the mouse ovary. Cell. Tiss. Kinet., 3, 1-11.

INGRAM D. L., 1962. Atresia, 247-273. In ZUCKERMAN S., The ovary, vol. 1, Acad. Press.

JEPPESEN Th., 1978. The ulirastructure of follicle cells in fetal guinea pig ovaries. Anat. Rec., 189, 649-668.

KENNELY J. J., FOOTE R. H., 1966. Oocytogenesis in rabbit. The role of neogenesis in the formafion of the definitive ova and the stability of oocyte DNA measured with tritiated thymidine. Am. J. Anat., 118, 573-590.

MARIANA J. C., MILLIER C., 1977. Application de quelques modèles de dynamique des populations à l'étude de la folliculogenèse ovarienne. Ann. Biol. anim. Bioch. Biophys., 17, 193-206.

MAULEON P., 1975. Importance des différentes périodes ovogénétiques dans la gonade femelle d'embryon de brebis ; contrôle du changement de comportement mitotique en méiotique. Ann. Biol. anim. Bioch. Biophys., 15, 725-738.

MENDELSOHN M. L., 1962. Autoradiographic analysis of cell proliferation in spontaneous Breast cancer of $\mathrm{C} 3 \mathrm{H}$ Mouse. III. The growth fraction J. nat. Cancer Inst., 28, 1015-1029.

PEDERSEN T., 1970. Follicle kinetics in the ovary of the cyclic mouse. Acta endocr., 64, 304-323.

PEDERSEN T., HARTMAN N. R., 1971. The kinetics of granulosa cells in developing follicles in the mouse ovary. Cell. Tiss. Kinet., 4, 171-184.

PETERS H., LEVY E., 1966. Cell dynamics of the ovarian cycle. J. Reprod. Fert., 11, 227-236.

PETERS H., BYSKOV A. G., HIMMELSTEIN-BRAW R., FABER M., 1975. Follicular growth : the basic event in the mouse and human ovary. J. Reprod. Fert., 45, 559-566. 